\title{
Avaliação genética de caprinos da raça Alpina utilizando-se a produção de leite no dia do controle
}

\author{
José Lindenberg Rocha Sarmento', João Cruz Reis Filho1, Lucia Galvão de Albuquerque², Paulo Sávio Lopes³, \\ Marcelo Teixeira Rodrigues ${ }^{3}$
}

${ }^{1}$ Pós-Graduação em Genética e Melhoramento Animal/UFV. Bolsista da CAPES.
2 Departamento de Zootecnia - Universidade Estadual Paulista.
${ }^{3}$ Departamento de Zootecnia - Universidade Federal de Viçosa. Bolsista do CNPq.

RESUMO - Com os objetivos de estimar os parâmetros genéticos e predizer os valores genéticos para produção de leite no dia do controle (PLDC) e para produção acumulada (PAC), utilizaram-se dados de 356 cabras da raça Alpina, com 825 lactações e 26.389 controles semanais efetuados entre os anos de 1997 e 2004. A PLDC foi analisada considerando-se o efeito fixo de grupo contemporâneo (ano-mês de controle), a idade da cabra ao parto, como covariável, e os efeitos aleatórios genético aditivo e de ambiente permanente. Para PAC, o modelo incluiu os mesmos efeitos aleatórios, o efeito fixo de grupo contemporâneo (ano-mês de parto) e as covariáveis idade da cabra ao parto e duração da lactação. Em geral, as herdabilidades estimadas foram de maiores magnitudes na primeira lactação, nos controles intermediários, indicando possibilidade de resposta à seleção. As correlações de ordem entre os valores genéticos para a produção até 305 dias de lactação e as PLDC intermediárias foram maiores que as do início e do final da lactação. Apesar de a correlação de ordem ter sido alta, houve alterações na classificação dos reprodutores para produção até 305 dias de lactação quando a seleção foi praticada com base na PLDC aos 90 dias. A seleção pela PLDC e PAC no meio da lactação pode ser indicada como critério de seleção, em substituição à produção até 305 dias.

Palavras-chave: caprinos leiteiros, componentes de variância, modelo animal, parâmetros genéticos, seleção

\section{Genetic evaluation of Alpine goats using test day milk yield}

\begin{abstract}
Data consisting of 26,389 individual test day milk yield from 825 lactations of 356 Parda Alpina goats, recorded from 1997 to 2004, were used to estimate genetic parameters and to predict breeding values for test day (PLDC) and accumulated (PAC) milk yields. The model for PLDC included the fixed effects of contemporary group (year-month of test), age of dam at kidding as covariate, and additive genetic, permanent environment and residual random effects. The model for PAC included the same random effects used for PLDC and the fixed effects of contemporary group (year-month of kidding) and the covariates age of dam at kidding and lactation length. Larger heritability estimates for milk yield in the intermediary test days indicate possibility of selection response. Rank correlation between breeding values for milk yield up to 305 days and intermediary PLDC were larger than those on early and late lactation. Despite the high rank correlation, changes in ranking of bucks for milk yield up to 305 days were observed when selection was based on PLDC at 90 days. Selection based on PLDC and PAC in middle lactation may be used as a selection criterion in substitution for milk yield up to 305 days.
\end{abstract}

Key Words: animal model, dairy goats, genetic parameters, seletion, variance components

\section{Introdução}

Nos últimos anos, a caprinocultura leiteira tem crescido significativamente, como conseqüência da iniciativa de produtores com maior visão empresarial e de programas governamentais, como ocorre no Rio Grande do Norte e na Paraíba. Entretanto, na área de melhoramento genético, o caprino tem recebido pouca atenção, se comparado a outras espécies, representando um desafio constante aos pesquisadores.

As análises de dados da produção de leite durante a vida da cabra podem ser conduzidas de várias formas. A mais simples seria por meio dos modelos de repetibilidade, em que a produção de leite nos diferentes períodos da lactação seria considerada medida repetida da característica, tendo como pressuposição homogeneidade de variâncias genéticas e não-genéticas ao longo da lactação, o que não é verdadeiro. Outra possibilidade seriam os modelos de dimensão finita ou aqueles que consideram a produção de leite no dia de controle (PLDC), denominados “test day models", em análises uni ou multicaracterísticas.

Os modelos PLDC apresentam algumas vantagens sobre o modelo tradicional de análise da produção total na 
lactação, pois permitem quantificar fatores específicos de cada dia de controle, que podem mudar entre os animais e de um controle para outro, e controlar o efeito de ambiente com maior precisão(Ptack \& Schaeffer, 1993). Outra vantagem é o fato de dispensar o uso de projeção de lactações parciais para produção total (El Faro \& Albuquerque, 2005), visto que os fatores de projeção assumem curva de lactação padrão. Desse modo, as produções serão subestimadas nos animais com maior persistência de lactação e superestimadas naqueles com menor persistência de lactação (Jamrozik \& Schaeffer, 1997). Ao utilizar esses fatores de ajuste, elimina-se a existência de variabilidade na forma da curva de lactação de cada animal e, conseqüentemente, alguma variação genética para a produção. Outro ponto importante é a diminuição de viés na estimação dos componentes de variância e na predição dos valores genéticos (Swalve, 1995).

O conhecimento dos parâmetros genéticos e fenotípicos das características utilizadas como critérios de seleção e a avaliação genética são indispensáveis para escolha dos pais da futura geração (Tholon et al., 2001). Uma vez que esta técnica permite a avaliação dos animais antes do encerramento das lactações, poderia se praticar seleção precocemente e com maior acurácia, o que proporcionaria diminuição no intervalo de gerações e maximização do ganho genético por unidade de tempo. Além disso, o emprego desse método não exige mudanças no sistema de controle leiteiro; ao contrário, as informações coletadas são mais eficientemente aproveitadas.

Os modelos que consideram a PLDC têm sido pouco utilizados para avaliação genética em caprinos. Na literatura consultada, constam relatos da utilização deste método para avaliação genética no Canadá desde 1999. Segundo Sullivan \& Wiggans (2000), em 1999, as avaliações foram feitas com base em 62.018 registros de PLDC, referentes a 11.778 lactações de 6.636 fêmeas.

Não foram encontrados relatos da estimação de parâmetros genéticos para PLDC em caprinos no Brasil. As estimativas obtidas são para produção total na lactação, como trabalhos realizados por Soares Filho et al. (2001), Tholon et al. (2001), Gonçalves et al. (2002) e Pimenta Filho et al. (2004), que apresentaram estimativas de herdabilidade de 0,$29 ; 0,37 ; 0,06$ e 0,23 , respectivamente.

Objetivou-se com este trabalho estimar os parâmetros genéticos e predizer os valores genéticos para produção de leite no dia do controle e para produção acumulada em diferentes períodos e estudar critérios de seleção com base nessas produções, em substituição à produção total na lactação, para cabras da raça Alpina.

\section{Material e Métodos}

Neste estudo, utilizaram-se 26.389 controles referentes a 825 lactações de 356 cabras da raça Alpina, controladas entre 1997 e 2004, pertencentes ao rebanho caprino do Departamento de Zootecnia da Universidade Federal de Viçosa, em Viçosa - MG. Os animais foram mantidos em baias coletivas sob o sistema de estabulação livre e receberam alimentação à base de silagem de milho e feno como volumoso e mistura concentrada, fornecida conforme a necessidade dos animais. Os controles foram registrados semanalmente, por meio de ordenha mecânica, realizada duas vezes ao dia.

As lactações foram truncadas aos 305 dias e divididas em nove períodos de 30 dias, que constituíram diferentes características (Tabela 1). Foram mantidas no arquivo de análise cabras com até quatro lactações, exigindo-se também pelo menos seis controles por lactação e que o primeiro tenha sido até 60 dias do seu início. Foram eliminados da análise animais com idade ao primeiro parto superior a 36 meses e aqueles em grupos contemporâneos com menos de dois animais.

Além das PLDC, utilizaram-se também as produções acumuladas de leite (PAC) a cada 30 dias, dos 60 aos 305 dias, assumidas como diferentes características. As PAC foram calculadas pelo método escada corrigida, adaptado de Bianchini Sobrinho (1984):

$$
P_{i}=Y_{1} X_{1}+\sum_{j=1}^{n} y_{i} x_{i}
$$

em que $\mathrm{P}_{\mathrm{i}}=$ produção de leite acumulada até o i-ésimo controle de lactação, sendo $\mathrm{i}=60,90,120,150,180,210,240$, 270 e 305 dias de lactação; $Y_{1}=$ produção de leite no primeiro controle; $\mathrm{X}_{1}=$ intervalo do início da lactação ao primeiro controle; $\mathrm{n}=$ número de controles; $\mathrm{y}_{\mathrm{i}}=$ produção de leite no $\mathrm{j}$-ésimo controle; e $\mathrm{x}_{\mathrm{i}}=$ intervalo dos controles.

As características foram analisadas por meio de um modelo animal, em análises unicaracterísticas, considerando-se apenas a primeira e todas as lactações em conjunto. Os modelos estatísticos utilizados para análise das PLDC para todas as lactações incluíram os efeitos genético aditivo direto e de ambiente permanente, como aleatórios, o efeito fixo de grupo contemporâneo, formado pelo ano e mês do controle, e a idade da cabra ao parto, como covariável regressões linear e quadrática.

Para a análise das PAC, consideraram-se os mesmos efeitos aleatórios e o efeito fixo de grupo contemporâneo, que, neste caso, foi formado pelo ano e mês do parto. Além da covariável idade da cabra ao parto, como regressões linear e quadrática, considerou-se também a duração da 
Tabela 1 - Número de observações, médias, desvios-padrão e coeficientes de variação (CV) da produção de leite, de acordo com o mês do controle e a produção até 305 dias de lactação, considerando-se a primeira e todas as lactações

Table 1 - Number of records, means, standard deviations and coefficients of variation (CV) for milk yield according to month of control and milk yield until 305 days considering the first and all lactations

\begin{tabular}{lcccc}
\hline Controle & Dias em lactação & No de observações & Médias (kg) & Desvios-padrão (kg) \\
Control & Days in milk & Number of records & Means (kg) & Standard deviation (kg) \\
\hline
\end{tabular}

Primeira lactação First lactation

$\begin{array}{lr}30 & 23-37 \\ 60 & 53-67 \\ 90 & 83-97 \\ 120 & 113-127 \\ 150 & 143-157 \\ 180 & 173-187 \\ 210 & 203-217 \\ 240 & 233-247 \\ 270 & 263-277 \\ 305^{*} & \end{array}$

229

269

251

210

205

185

160

141

247

305

\section{0}

60

90

120

150

180

210

240

270

$305^{*}$

$23-37$
$53-67$
$83-97$
$113-127$
$143-157$
$173-187$
$203-217$
$233-247$
$263-277$

23-37

83-97

$173-187$

$233-247$
Produção acumulada até 305 dias de lactação.

* Milkyield until 305 days.

lactação, como regressão de mesma ordem. Uma vez que existem relatos na literatura, em bovinos leiteiros, de que o ajuste para duração da lactação diminui a variabilidade genética para produção de leite (Mello et al., 1994), pela alta correlação entre elas, definiram-se duas estratégias de análises, incluindo ou não a duração da lactação no modelo estatístico, de modo a investigar essa situação em caprinos leiteiros.

Matricialmente, o modelo estatístico pode ser representado como a seguir:

$$
y=X b+Z_{1} a+Z_{2} e p+\varepsilon
$$

em que $\mathrm{y}=\mathrm{o}$ vetor da PLDC ou PAC; $\mathrm{b}=\mathrm{o}$ vetor de efeitos fixos, contendo grupo contemporâneo e covariáveis; $\mathrm{a}=\mathrm{o}$ vetor de efeitos genéticos aditivos diretos; ep = o vetor de efeitos de ambiente permanente; $\mathrm{X}, \mathrm{Z}_{1} \mathrm{e} \mathrm{Z}_{2}=$ as matrizes de incidência que relacionam as observações aos efeitos fixos, genéticos aditivos diretos e de ambiente permanente, respectivamente; e $\varepsilon=$ o vetor de resíduos aleatórios.

As pressuposições assumidas em relação aos vetores a, ep e $\varepsilon$ são as que possuem distribuição normal, com E(a) $=\mathrm{E}(\mathrm{ep})=\mathrm{E}(\varepsilon)=0$ e $\operatorname{Var}(\mathrm{a})=A \otimes \sigma_{a}^{2}, \operatorname{Var}(\mathrm{ep})=I_{n} \otimes \sigma_{e p}^{2} \mathrm{e}$ $\operatorname{Var}(\varepsilon)=I_{N} \otimes \sigma_{e}^{2}$, sendo $\sigma_{a}^{2}, \sigma_{e p}^{2}$ e $\sigma_{e}^{2}$ as variâncias genética aditiva direta, de ambiente permanente e residual, respectivamente; A, a matriz de numeradores dos coeficientes de parentesco de Wright entre os animais; $\mathrm{I}_{\mathrm{n}}$, uma matriz identidade de ordem $\mathrm{n}$, sendo $\mathrm{n}$ o número de animais com observações; $\mathrm{I}_{\mathrm{N}}$, uma matriz identidade de ordem $\mathrm{N}$, sendo N o número de observações; e $\otimes$, o produto de Kroenecker entre matrizes.

Para as análises da primeira lactação, os mesmos modelos foram empregados, exceto o efeito aleatório de ambiente permanente, que foi desconsiderado, visto que, na primeira lactação, não há medidas repetidas.

Os componentes de variância foram estimados pelo método da Máxima Verossimilhança Restrita, utilizando-se um algoritmo livre de derivadas, disponível no programa MTDFREML (Boldman et al., 1995).

Os valores genéticos foram preditos para todas as características na primeira e em todas as lactações conjuntamente. Posteriormente, estimaram-se as correlações de ordem e amostrais entre os valores genéticos preditos para as PLDC e PAC, no intuito de averiguar o que ocorreria com a classificação dos animais para produção até 305 dias de lactação quando a seleção fosse praticada nas PLDC e PAC.

\section{Resultados e Discussão}

As PLDC apresentaram altos CV (Tabela 1), os quais tenderam a crescer do início para o final da lactação, podendo ser indicativo de variação na forma da curva de lactação das 
cabras desse rebanho, tanto na primeira quanto em todas as lactações. A maior variação da produção no final da lactação era, de certa forma, esperada, uma vez que o número de observações diminuiu quando se aproximou o final da lactação das cabras. A produção de leite na primeira lactação variou conforme uma curva padrão de lactação para raças especializadas, apresentando um pico de produção em torno dos 60 dias de lactação (Tabela 1).

As variâncias fenotípicas (Tabela 2), tanto na primeira como em todas as lactações, foram maiores no início da lactação, com pico aos 60 dias, tendendo a diminuir com o decorrer da lactação, exceto ao final, em que houve ligeiro aumento, provavelmente em razão do menor número de informações. O mesmo comportamento foi verificado para a variância residual.

As variâncias genéticas aditivas (Tabela 2) foram bem menores que as residuais na primeira e em todas as lactações, entretanto, as estimadas para a primeira lactação foram ligeiramente maiores, no início e meio da lactação, que as estimadas para todas as lactações. As variâncias estimadas no final da lactação foram baixas, próximas de zero, provavelmente em razão do baixo número de informações. As maiores variâncias genéticas aditivas para a primeira lactação são coerentes, uma vez que na primeira lactação costuma-se mensurar a produção de todas as cabras, tendo como conseqüência maior variabilidade. Além disso, normalmente apenas as melhores cabras continuam sendo controladas nas lactações posteriores, resultando em maior homogeneidade, e conseqüentemente, em menor variabilidade genética.

As variâncias genéticas aditivas na primeira lactação apresentaram comportamento irregular, com tendência de variâncias estimadas para os controles do meio da lactação serem ligeiramente maiores. Conseqüentemente, as estimativas de herdabilidade apresentaram o mesmo comportamento, confirmando a maioria dos resultados encontrados na literatura para bovinos leiteiros (Jamrozik \& Schaeffer, 1997; Ferreira et al., 2003; Mello et al., 2005). Quando todas as lactações foram utilizadas, o comportamento das variâncias genéticas foi diferente, sendo menores do meio para o final, com exceção da produção aos 270 dias, que foi maior, resultando em mesmo comportamento para as herdabilidades.

A variância para o efeito de ambiente permanente aumentou até 120 dias de lactação, apresentou menor valor aos 150 dias, elevou ligeiramente aos 180 dias e, a partir daí, permaneceu quase constante até o final da lactação. Tendência semelhante foi verificada para a repetibilidade, que aumentou até o controle aos 120 dias, atingindo o máximo de 0,45 , seguido por um comportamento irregular, variando de 0,26 a 0,40 até aos 270 dias (Tabela 2). De modo geral, a repetibilidade para a PLDC apresentou variação de baixa à média magnitude, de modo que uma única medida no animal pode não representar a real capacidade de produção, indicando que são necessárias mais de uma medida para aumentar a precisão das estimativas, refletindo em maior confiabilidade nas inferências a serem realizadas.

As estimativas de herdabilidade oscilaram ao longo da curva de lactação, variando de 0,05 a 0,43 e de 0,07 a 0,27, respectivamente, na primeira e em todas as lactações (Tabela 2). De modo geral, as herdabilidades na primeira foram superiores às suas correspondentes, estimadas para todas as lactações. Na primeira lactação, as herdabilidades estimadas diminuíram dos 30 aos 90 dias, período próximo ao pico de produção. A partir daí, tenderam a valores maiores até 210 dias de lactação, voltando a cair até os 270 dias. Considerando-se todas as lactações, houve também tendência de maiores valores de herdabilidade para os controles intermediários.

De maneira geral, as herdabilidades estimadas para PLDC foram próximas às encontradas na literatura nacional para produção total na lactação (Tholon et al., 2001; Gonçalves et al., 2002; Pimenta Filho et al., 2004), de modo que as estimadas para as produções de controles intermediárias, na primeira lactação, tenderam a ser superiores. $\mathrm{Na}$ literatura internacional, poucos trabalhos têm apresentado estimativas de herdabilidade para PLDC de cabras. Breznik et al. (2000), ao estimarem componentes de variância para PLDC utilizando um modelo de repetibilidade, em cabras da Slovenia, encontraram herdabilidade igual a 0,20.

As análises das PAC foram realizadas incluindo ou não a duração da lactação no modelo (resultados não apresentados). Quando a duração foi considerada, constatou-se aumento nas herdabilidades conforme a diminuição das variâncias residuais, contrariando os resultados obtidos por Mello et al. (1994), que verificaram que a inclusão da duração da lactação no modelo reduziu a variação genética, e não a residual. Desta forma, os resultados apresentados para as produções acumuladas são os obtidos pelo modelo estatístico que corrigiu a duração da lactação (Tabela 3).

As variâncias estimadas para as PAC cresceram linearmente conforme os dias em lactação, tanto na primeira quanto em todas as lactações. Da mesma forma que nas análises para PLDC, as variâncias fenotípicas e residuais para as produções acumuladas foram de magnitudes maiores para todas as lactações que para a primeira lactação. As variâncias genéticas aditivas, no entanto, foram de maiores magnitudes na primeira lactação, refletindo em maiores herdabilidades para as PAC na primeira lactação. 
Tabela 2 - Estimativas de variâncias ${ }^{*}$, herdabilidades $\left(\mathrm{h}^{2}\right)$ e repetibilidade (t) para as produções no dia de controle na primeira e em todas as lactações

Table 2 - Estimates of variances ${ }^{*}$, heritabilities $\left(h^{2}\right)$ and repeatability $(t)$ for test-day milk yields on first and all lactations

\begin{tabular}{lcccc}
$\begin{array}{l}\text { Controle } \\
\text { Control }\end{array}$ & $\sigma_{a}^{2}$ & $\sigma_{e p}^{2}$ & $\sigma_{e}^{2}$ & $\sigma_{p}^{2}$ \\
\hline
\end{tabular}

Primeira lactação

First lactation

\begin{tabular}{|c|c|c|c|c|c|c|}
\hline 30 & 0,1911 & - & 0,4777 & 0,6689 & 0,29 & - \\
\hline 60 & 0,1624 & - & 0,5708 & 0,7333 & 0,22 & - \\
\hline 90 & 0,1213 & - & 0,5647 & 0,6861 & 0,18 & - \\
\hline 120 & 0,2271 & - & 0,4844 & 0,7116 & 0,32 & - \\
\hline 150 & 0,2374 & - & 0,3524 & 0,5900 & 0,40 & - \\
\hline 180 & 0,1441 & - & 0,3092 & 0,4533 & 0,32 & - \\
\hline 210 & 0,1901 & - & 0,2512 & 0,4414 & 0,43 & - \\
\hline 240 & 0,0559 & - & 0,3211 & 0,3770 & 0,15 & - \\
\hline & \multicolumn{6}{|c|}{$\begin{array}{c}\text { Todas as lactações } \\
\text { All lactations }\end{array}$} \\
\hline 30 & 0,0797 & 0,1668 & 0,7182 & 0,9648 & 0,08 & 0,25 \\
\hline 60 & 0,1593 & 0,1716 & 0,7741 & 1,1051 & 0,14 & 0,30 \\
\hline 90 & 0,1615 & 0,2600 & 0,5612 & 0,9828 & 0,16 & 0,42 \\
\hline 120 & 0,1047 & 0,2952 & 0,4932 & 0,8932 & 0,12 & 0,45 \\
\hline 150 & 0,1220 & 0,0676 & 0,5134 & 0,7032 & 0,17 & 0,26 \\
\hline 210 & 0,0656 & 0,1155 & 0,3037 & 0,4849 & 0,14 & 0,38 \\
\hline 240 & 0,0313 & 0,1007 & 0,3330 & 0,4651 & 0,07 & 0,29 \\
\hline 270 & 0,1472 & 0,0683 & 0,3282 & 0,5437 & 0,27 & 0,40 \\
\hline
\end{tabular}

*Variâncias $\left(\mathrm{kg}^{2}\right)$ : genéticas aditivas $\left(\sigma_{a}^{2}\right)$, de ambiente permanente $\left(\sigma_{e p}^{2}\right)$, residuais $\left(\sigma_{e}^{2}\right)$ e fenotípicas $\left(\sigma_{p}^{2}\right)$.

${ }^{*}$ Variances $\left(\mathrm{kg}^{2}\right)$ : additive genetic $\left(\sigma_{a}^{2}\right)$, permanent environment $\left(\sigma_{e p}^{2}\right)$, residual $\left(\sigma_{e}^{2}\right)$ and phenotypic $\left(\sigma_{p}^{2}\right)$.

As variâncias de ambiente permanente (Tabela 3) apresentaram comportamento semelhante ao das variâncias genéticas aditivas, diferindo apenas na magnitude dos valores, que foram ligeiramente superiores às variâncias genéticas ao longo de toda lactação, indicando variação decorrente de causas ambientais permanentes entre os indivíduos.

As herdabilidades estimadas para as PAC foram de maiores magnitudes que as estimadas para as PLDC (Tabelas 2 e 3). Da mesma forma que para as PLDC, as herdabilidades estimadas para as PAC do meio tenderam a ser mais elevadas que as estimadas no início e no final da lactação. As diferenças são, sobretudo, pelo fato de as estimativas obtidas para as PLDC terem oscilado mais ao longo da lactação.

As correlações de ordem e amostrais entre os valores genéticos preditos para as PLDC e para produção até 305 dias de lactação encontram-se na Tabela 4. De modo geral, ambas as correlações foram maiores entre os valores genéticos preditos para as PLDC adjacentes e menores à medida que os controles se distanciaram. As maiores correlações foram obtidas entre os valores genéticos para as PLDC do meio da lactação $(90,120,150$ e 180 dias), enquanto as menores foram entre os valores genéticos preditos para as do início e do final da lactação. As correlações estimadas entre os valores genéticos preditos, considerando todas as lactações, apresentaram o mesmo comportamento das estimadas para a primeira, porém, foram de maiores magnitudes.

As estimativas de correlações entre os valores genéticos preditos para PLDC e a produção acumulada até 305 dias foram maiores com os controles do meio da lactação, sendo que, na primeira lactação, a maior correlação, seja de ordem seja amostral, foi obtida com a PLDC aos 90 dias, igual a 0,77 (Tabela 4). Quando consideradas todas as lactações, esta correlação foi superior a 0,80. As correlações entre os valores genéticos das produções nos controles de 90 a 180 dias e a produção acumulada até 305 dias foram todas superiores a 0,80 . Correlação de 0,62 entre os valores genéticos preditos para produção de leite até 305 dias e para PLDC foi obtida por Ferreira et al. (2003), que analisaram primeiras lactações de bovinos da raça Holandesa e concluíram que é viável a utilização da PLDC em substituição à produção até 305 dias de lactação na avaliação genética de vacas e touros. Porém, recomendaram que apenas os controles do meio da lactação fossem utilizados como critério de seleção, pois foram mais correlacionados à produção até 305 dias.

As correlações estimadas entre os valores genéticos preditos para as PAC (Tabela 5) apresentaram o mesmo comportamento das estimadas para as PLDC, que, como 
Tabela 3 - Estimativas de variâncias*, herdabilidade $\left(\mathrm{h}^{2}\right)$ e repetibilidade $(\mathrm{t})$ para as produções parciais acumuladas na primeira e em todas as lactações

Table 3 - Estimates of variances ${ }^{*}$, heritabilities $\left(h^{2}\right)$ and repeatability $(t)$ for accumulated milk partial yields on first and all lactations

\begin{tabular}{lcccc}
$\begin{array}{l}\text { Período } \\
\text { Period }\end{array}$ & $\sigma_{a}^{2}$ & $\sigma_{e p}^{2}$ & $\sigma_{e}^{2}$ & $\sigma_{p}^{2}$ \\
\hline
\end{tabular}

Primeira lactação

First lactation

$\begin{array}{ll}60 & 428,58 \\ 90 & 1488,62 \\ 120 & 2594,46 \\ 150 & 3875,02 \\ 180 & 4932,55 \\ 210 & 5001,64 \\ 240 & 5569,32 \\ 270 & 6053,13 \\ 305 & 5402,83\end{array}$

$\begin{array}{lcl}1031,23 & 1459,81 & 0,29 \\ 2151,32 & 3639,94 & 0,41 \\ 3765,30 & 6359,77 & 0,41 \\ 4894,11 & 8769,13 & 0,44 \\ 5845,43 & 10777,98 & 0,46 \\ 6973,66 & 11975,31 & 0,42 \\ 7471,28 & 13040,61 & 0,43 \\ 8164,02 & 14217,16 & 0,43 \\ 9641,01 & 15043,83 & 0,36\end{array}$

Todas as lactações

All lactations

\begin{tabular}{|c|c|c|c|c|c|c|}
\hline 60 & 303,90 & 464,51 & 1475,34 & 2243,75 & 0,14 & 0,35 \\
\hline 90 & 838,76 & 1038,91 & 3203,62 & 5081,30 & 0,17 & 0,37 \\
\hline 120 & 1688,57 & 1760,86 & 4949,55 & 8398,98 & 0,20 & 0,41 \\
\hline 150 & 2447,77 & 2982,63 & 6282,32 & 11712,73 & 0,21 & 0,46 \\
\hline 180 & 3039,08 & 3679,09 & 7999,70 & 14717,87 & 0,21 & 0,46 \\
\hline 210 & 3315,57 & 4524,63 & 9123,96 & 16964,17 & 0,20 & 0,47 \\
\hline 240 & 3634,12 & 5097,76 & 10161,23 & 18893,12 & 0,19 & 0,46 \\
\hline 270 & 3986,68 & 5020,29 & 10986,10 & 19993,07 & 0,20 & 0,45 \\
\hline 305 & 4139,57 & 5186,93 & 12059,16 & 21385,66 & 0,19 & 0,43 \\
\hline
\end{tabular}

* Variâncias $\left(\mathrm{kg}^{2}\right)$ : genéticas aditivas $\left(\sigma_{a}^{2}\right)$, de ambiente permanente $\left(\sigma_{e p}^{2}\right)$, residuais $\left(\sigma_{e}^{2}\right)$ e fenotípicas $\left(\sigma_{p}^{2}\right)$.

*Variances $\left(\mathrm{kg}^{2}\right)$ : additive genetic $\left(\sigma_{a}^{2}\right)$, permanent environment $\left(\sigma_{e p}^{2}\right)$, residual $\left(\sigma_{e}^{2}\right)$ and phenotypic $\left(\sigma_{p}^{2}\right)$.

esperado, foram de maiores magnitudes. As correlações entre os valores genéticos para as PAC e a produção acumulada até 305 dias foram crescentes dos 60 até os 270 dias de lactação, sendo que, aos 90 dias, foram de 0,80 para primeira e de 0,89 para todas as lactações.

A medida padrão de produção de leite para avaliação genética, até início da década de 90 , era a produção total na lactação (ou a produção acumulada até 305 dias de lactação, como tem sido freqüentemente utilizada em bovinos leiteiros). Nessa época, os controles de produções eram utilizados apenas para se calcular a produção total na lactação. Atualmente, a PLDC tem sido utilizada em substituição à produção total nas avaliações genéticas de caprinos leiteiros no Canadá (Sullivan \& Wiggans, 2000). A utilização deste método permite que animais com poucos controles possam ser avaliados, não sendo necessário aguardar o término da lactação, acelerando o processo de avaliação genética.

Avaliaram-se as classificações dos bodes com base nos valores genéticos preditos para PLDC e PAC aos 90 dias, em relação à produção acumulada até 305 dias de lactação, considerando apenas a primeira e todas as lactações (Figura 1). Observou-se consistência na classificação dos reprodutores utilizando-se apenas a primeira ou todas as lactações, ou seja, os que foram melhores ou piores em uma situação também o foram na outra. Apesar da alta correlação entre os valores genéticos, houve alteração na classificação, ocorrendo uma situação em que um reprodutor foi classificado negativamente para PLDC e positivamente para produção acumulada até 305 dias de lactação. Entretanto, quando se observou a classificação com base na produção PAC até 90 dias, constataram-se menores alterações em comparação à PLDC aos 90 dias de lactação. Esses resultados são válidos, uma vez que a correlação da PAC até 90 dias foi superior à obtida com a PLDC.

De modo geral, maiores ganhos podem ser obtidos quando a seleção é praticada utilizando-se os controles próximos ao meio da lactação, uma vez que estes são mais estáveis e mais correlacionados à produção até 305 dias. Entretanto, em razão das alterações na classificação dos bodes, esses resultados devem ser vistos com certa cautela, uma vez que o número de animais avaliados foi pequeno. Há necessidade de estudos complementares envolvendo maior volume de dados, o que apenas será possível quando houver, por parte dos criadores de caprinos, a conscientização de que fazer controle leiteiro é fundamental. Além disso, seria essencial a realização de estudos econômicos, de modo que fosse determinado o impacto econômico em adotar, como critério de seleção, PLDC do 
Tabela 4 - Correlações de ordem (acima da diagonal) e amostrais (abaixo da diagonal) entre os valores genéticos preditos para as produções no dia de controle e até 305 dias de lactação na primeira e em todas as lactações

Table 4 - Rank (above diagonal) and sample (below diagonal) correlations between predict breeding values for test-day and until 305 days milk yields on first and all lactations

\begin{tabular}{lllllllll}
\hline $\begin{array}{l}\text { Controle } \\
\text { Control }\end{array}$ & 30 & 60 & 90 & 120 & 150 & 180 & 210 & 240 \\
\hline
\end{tabular}

\begin{tabular}{|c|c|c|c|c|c|c|c|c|c|c|}
\hline \multicolumn{11}{|c|}{$\begin{array}{l}\text { Primeira lactação } \\
\text { First lactation }\end{array}$} \\
\hline 30 & 1 & 0,65 & 0,60 & 0,56 & 0,51 & 0,39 & 0,43 & 0,44 & 0,35 & 0,53 \\
\hline 60 & 0,64 & 1 & 0,79 & 0,71 & 0,66 & 0,61 & 0,58 & 0,41 & 0,22 & 0,73 \\
\hline 90 & 0,60 & 0,79 & 1 & 0,73 & 0,64 & 0,55 & 0,52 & 0,48 & 0,27 & 0,77 \\
\hline 120 & 0,59 & 0,73 & 0,74 & 1 & 0,73 & 0,70 & 0,62 & 0,31 & 0,21 & 0,71 \\
\hline 150 & 0,52 & 0,65 & 0,63 & 0,73 & 1 & 0,72 & 0,59 & 0,37 & 0,26 & 0,76 \\
\hline 180 & 0,43 & 0,62 & 0,56 & 0,71 & 0,73 & 1 & 0,63 & 0,34 & 0,17 & 0,71 \\
\hline 210 & 0,44 & 0,58 & 0,54 & 0,65 & 0,60 & 0,66 & 1 & 0,43 & 0,44 & 0,67 \\
\hline 240 & 0,46 & 0,47 & 0,52 & 0,35 & 0,38 & 0,38 & 0,49 & 1 & 0,62 & 0,54 \\
\hline 270 & 0,39 & 0,31 & 0,41 & 0,33 & 0,34 & 0,33 & 0,54 & 0,71 & 1 & 0,41 \\
\hline $305^{*}$ & 0,55 & 0,74 & 0,77 & 0,72 & 0,75 & 0,72 & 0,69 & 0,57 & 0,55 & 1 \\
\hline \multicolumn{11}{|c|}{$\begin{array}{c}\text { Todas as lactações } \\
\text { All lactations }\end{array}$} \\
\hline 30 & 1 & 0,80 & 0,69 & 0,70 & 0,61 & 0,63 & 0,58 & 0,62 & 0,37 & 0,74 \\
\hline 60 & 0,82 & 1 & 0,83 & 0,83 & 0,74 & 0,63 & 0,58 & 0,62 & 0,37 & 0,74 \\
\hline 90 & 0,71 & 0,84 & 1 & 0,82 & 0,77 & 0,76 & 0,61 & 0,64 & 0,39 & 0,86 \\
\hline 120 & 0,71 & 0,84 & 0,82 & 1 & 0,81 & 0,81 & 0,71 & 0,69 & 0,47 & 0,87 \\
\hline 150 & 0,64 & 0,76 & 0,79 & 0,83 & 1 & 0,85 & 0,68 & 0,66 & 0,48 & 0,81 \\
\hline 180 & 0,66 & 0,79 & 0,78 & 0,82 & 0,86 & 1 & 0,76 & 0,74 & 0,49 & 0,82 \\
\hline 210 & 0,58 & 0,66 & 0,60 & 0,71 & 0,68 & 0,76 & 1 & 0,71 & 0,62 & 0,71 \\
\hline 240 & 0,62 & 0,63 & 0,67 & 0,70 & 0,67 & 0,77 & 0,73 & 1 & 0,68 & 0,74 \\
\hline 270 & 0,38 & 0,36 & 0,40 & 0,50 & 0,48 & 0,51 & 0,62 & 0,67 & 1 & 0,55 \\
\hline $305^{*}$ & 0,77 & 0,84 & 0,87 & 0,88 & 0,83 & 0,84 & 0,72 & 0,76 & 0,57 & 1 \\
\hline
\end{tabular}

* Produção (Ver Tabela 1).

* Yield (See Table 1).

Tabela 5 - Correlações de ordem (acima da diagonal) e amostrais (abaixo da diagonal) entre os valores genéticos preditos para as produções acumuladas para cada período na primeira e em todas as lactações

Table 5 - $\quad$ Rank (above diagonal) and sample (below diagonal) correlations between predict breeding values for accumulated milk yields to each period on first and all lactations

\begin{tabular}{llllllll}
\hline $\begin{array}{l}\text { Período } \\
\text { Period }\end{array}$ & 60 & 90 & 120 & 150 & 180 & 210 & 240
\end{tabular}

\begin{tabular}{|c|c|c|c|c|c|c|c|c|c|}
\hline \multirow[b]{2}{*}{60} & \multicolumn{9}{|c|}{$\begin{array}{c}\text { Primeira lactação } \\
\text { First lactation }\end{array}$} \\
\hline & 1 & 0,95 & 0,92 & 0,88 & 0,83 & 0,81 & 0,78 & 0,76 & 0,76 \\
\hline 90 & 0,95 & 1 & 0,96 & 0,94 & 0,88 & 0,84 & 0,82 & 0,81 & 0,80 \\
\hline 120 & 0,93 & 0,97 & 1 & 0,96 & 0,93 & 0,89 & 0,86 & 0,85 & 0,84 \\
\hline 150 & 0,89 & 0,94 & 0,96 & 1 & 0,96 & 0,92 & 0,90 & 0,89 & 0,87 \\
\hline 180 & 0,84 & 0,88 & 0,92 & 0,96 & 1 & 0,95 & 0,92 & 0,91 & 0,89 \\
\hline 210 & 0,80 & 0,83 & 0,87 & 0,92 & 0,94 & 1 & 0,96 & 0,95 & 0,93 \\
\hline 240 & 0,77 & 0,81 & 0,85 & 0,90 & 0,92 & 0,96 & 1 & 0,97 & 0,96 \\
\hline 270 & 0,75 & 0,80 & 0,84 & 0,89 & 0,91 & 0,95 & 0,98 & 1 & 0,98 \\
\hline \multirow[t]{2}{*}{305} & 0,75 & 0,79 & 0,82 & 0,87 & 0,89 & 0,93 & 0,96 & 0,98 & 1 \\
\hline & \multicolumn{9}{|c|}{$\begin{array}{c}\text { Todas as lactações } \\
\text { All lactations }\end{array}$} \\
\hline 60 & 1 & 0,97 & 0,94 & 0,92 & 0,91 & 0,90 & 0,89 & 0,87 & 0,86 \\
\hline 90 & 0,97 & 1 & 0,98 & 0,96 & 0,95 & 0,94 & 0,93 & 0,91 & 0,89 \\
\hline 120 & 0,95 & 0,98 & 1 & 0,98 & 0,97 & 0,96 & 0,94 & 0,93 & 0,92 \\
\hline 150 & 0,93 & 0,96 & 0,98 & 1 & 0,99 & 0,98 & 0,97 & 0,96 & 0,95 \\
\hline 180 & 0,92 & 0,96 & 0,97 & 0,99 & 1 & 0,98 & 0,97 & 0,97 & 0,96 \\
\hline 210 & 0,91 & 0,94 & 0,96 & 0,98 & 0,99 & 1 & 0,99 & 0,98 & 0,97 \\
\hline 240 & 0,90 & 0,93 & 0,95 & 0,97 & 0,98 & 0,99 & 1 & 0,99 & 0,98 \\
\hline 270 & 0,88 & 0,92 & 0,94 & 0,96 & 0,97 & 0,98 & 0,99 & 1 & 0,99 \\
\hline 305 & 0,87 & 0,91 & 0,93 & 0,95 & 0,96 & 0,97 & 0,98 & 0,99 & 1 \\
\hline
\end{tabular}


a) Produção de leite no controle aos 90 dias (Test day milk yields at 90 days)
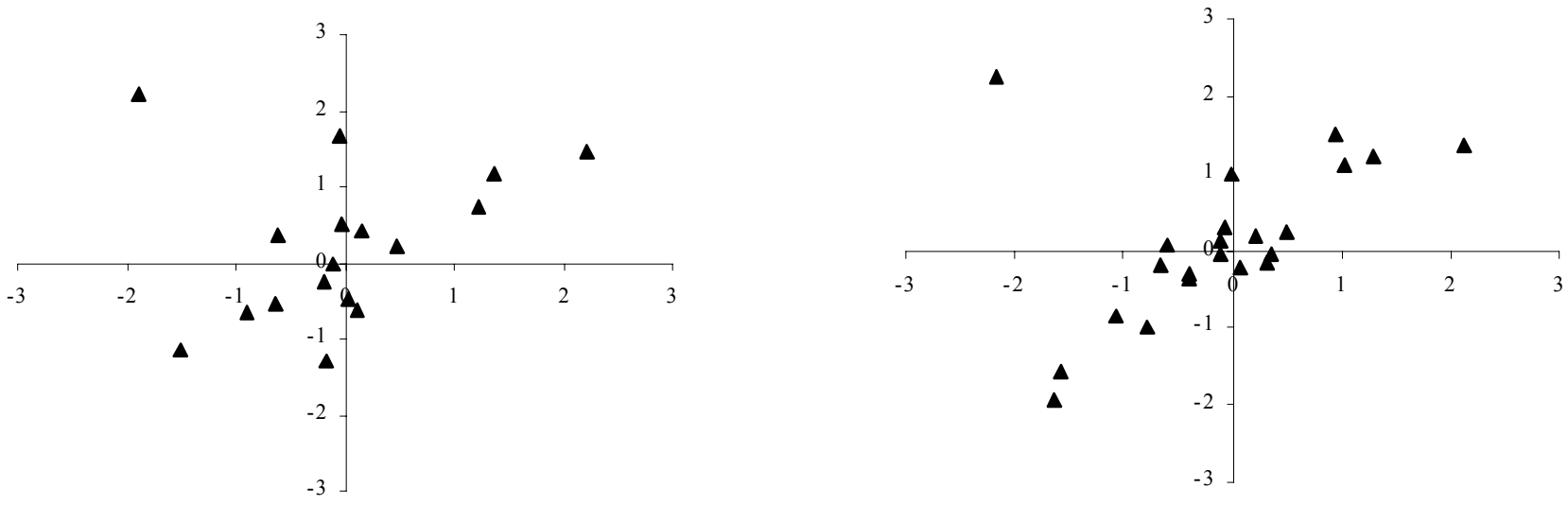

b) Produção acumulada até 90 dias (Acumulated milk yields until 90 days)

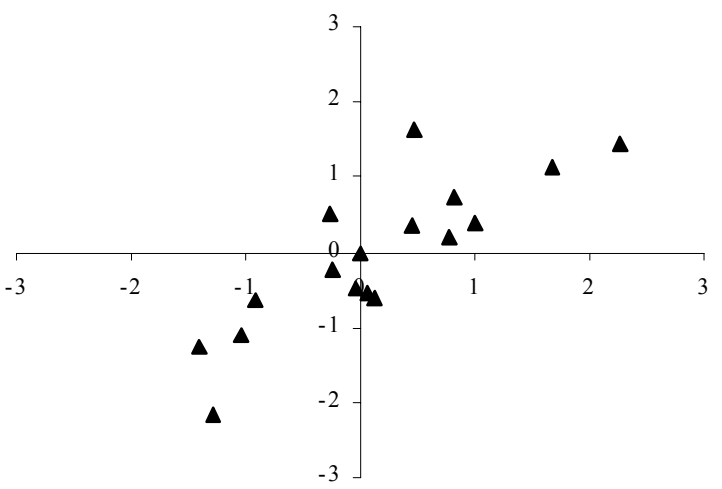

Primeira lactação (First lactation)

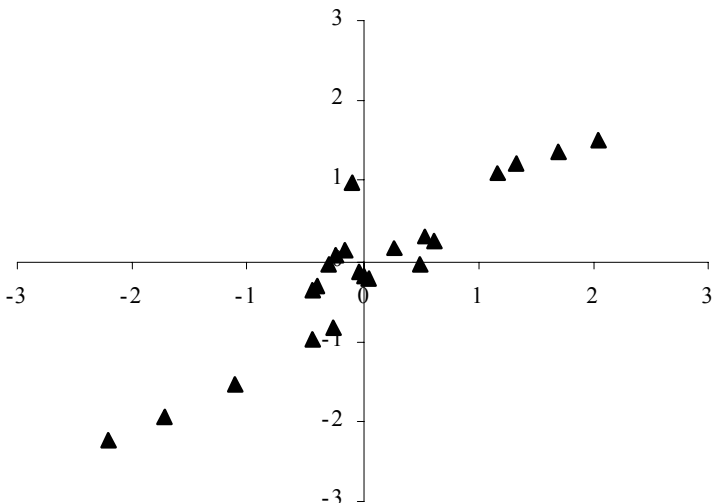

Todas as lactações (All lactations)

Figura 1 - Valores genéticos preditos dos bodes, em unidades de desvio-padrão, para produção de leite no dia de controle (a) e acumulada até 90 dias (b), eixo x, e a produção acumulada até 305 dias de lactação, eixo y, considerando-se a primeira (à esquerda) e todas as lactações (à direita).

Figure 1 - Bucks' predicted breeding values, in standard deviation units, fortest-day milk yields (a) and accumulated at 90 days (b), $x$ axis, and accumulated milk yields until 305 days, $y$ axis, considering the first (left) and all lactations (right).

meio da lactação em substituição à produção total ou acumulada até 305 dias de lactação. Diante do exposto, vale salientar que, apesar do pequeno número de informações utilizadas nesse estudo, os resultados obtidos são relevantes para a caprinocultura, em razão da escassez de trabalhos nesta linha na literatura nacional.

Uma vez constatada a possibilidade de se adotar PLDC como critérios de seleção, a utilização, por exemplo, da seleção para PLDC ou PAC aos 90 dias, ou próximo deste, poderia permitir selecionar os melhores reprodutores para produção acumulada até 305 dias de lactação, visto que foram altamente correlacionadas. Uma das vantagens em adotar este critério de seleção seria o fato de permitir avaliar as cabras mais precocemente, ou seja, não haveria a necessidade de se esperar que a cabra encerrasse a lactação para ser avaliada. A conseqüência imediata seria diminuição no intervalo de gerações, o que proporcionaria maior ganho genético por unidade de tempo. Além disso, a adoção deste método não requer mudança do sistema de controle leiteiro, que é bastante dispendioso; ao contrário, proporcionaria melhor aproveitamento das informações atualmente coletadas. Essas informações têm sido utilizadas apenas para se estimar a produção total na lactação, podendo-se visualizar um retorno econômico vantajoso, pois, com o mesmo investimento, os animais seriam avaliados com maior acurácia. 


\section{Conclusões}

As produções de leite no dia do controle no meio da lactação apresentaram maiores herdabilidades e variabilidades genéticas que as produções do início e do final da lactação, sugerindo que podem ser recomendadas na avaliação genética de caprinos leiteiros.

Considerando as altas correlações entre os valores genéticos para produções no dia do controle e acumuladas, no meio da lactação, e a produção acumulada até 305 dias de lactação, a PAC e PLDC podem ser indicadas como critério de seleção, em substituição à produção até 305 dias de lactação.

Outros estudos envolvendo conjuntos de dados mais representativos devem ser realizados, visando à confirmação e à validação das vantagens observadas neste estudo.

\section{Literatura Citada}

BIANCHINI SOBRINHO, E. Estudo da curva de lactação de vacas da raça Gir. Ribeirão Preto: Universidade de São Paulo, 1984. 88p. Tese (Doutorado em Genética) - Universidade de São Paulo, 1984

BOLDMAN, K.G.; KRIESE, L.A.; Van VLECK, D.L. et al. A manual for use of MTDFREML. A set of programs to obtain estimates of variances and covariances [DRAFT]. Lincon: USDA/ARS, 1995. $120 \mathrm{p}$.

BREZNIK, S.; MALOVRH, S.; KOVAC, M. et al. Additive genetic and environmental variance components for milk traits in goat with test day model. Zootehnika, v.76, n.1, p.61-66, 2000.

EL FARO, L.; ALBUQUERQUE, L.G. Predição de valores genéticos para a produção de leite no dia do controle e para a produçãoacumulada até 305 dias. Revista Brasileira de Zootecnia, v.34, n.2, p.496-507, 2005.

FERREIRA, W.J.; TEIXEIRA, N.M.; EUCLYDES, R.F. et al. Avaliação genética de bovinos da raça Holandesa usando a produção de leite no dia do controle. Revista Brasileira de Zootecnia, v.32, n.2, p.295-303, 2003.
GONCALVES, H.C.; SILVA, M.A.; WECHSLER, F.S. et al. Parâmetros e tendência genética da produção de leite de cabra no Brasil. Revista Brasileira de Zootecnia, v.31, n.6, p.2204$2208,2002$.

JAMROZIK, J.; SCHAEFFER, L.R. Estimates of genetic parameters for a test day model with random regression for yield traits. Journal of Dairy Science, v.80, p.762-770, 1997.

MELLO,A.A.; PENNA, V.M.; MADALENA, F.E. et al. Efeito da eliminação de lactações curtas e do ajuste pela duração da lactação na herdabilidade da produção de leite em um rebanho Gir. Archivos Latinoamericanos Producción Animal, v.2, p. 117-123, 1994.

MELLO, C.M.R.; PACKER, I.U.; COSTA, C.N.C. et al. Parâmetros genéticos para as produções de leite no dia do controle e da primeira lactação de vacas da raça Holandesa. Revista Brasileira de Zootecnia, v.34, n.3, p.796-806, 2005.

PIMENTA FILHO, E.C.; SARMENTO, J.L.R.; RIBEIRO, M.N. Efeitos genéticos e ambientais que afetam a produção de leite e duração da lactação de cabras mestiças no estado da Paraíba. Revista Brasileira de Zootecnia, v.31, n.6, p.1426-1431, 2004.

PTACK, E.; SCHAEFFER, L.R. Use of test day yields for genetic evaluation of dairy sires and cows. Livestock Production Science, v.34, p.23-34, 1993.

SOARES FILHO, G.; MCMANUS, C.; MARIANTE, A.S. Fatores genéticos e ambientais que influenciam algumas características de reprodução e produção de leite em cabras no Distrito Federal. Revista Brasileira de Zootecnia, v.30, n.1, p.53-59, 2001.

SULLIVAN, B.; WIGGANS, G. Genetic evaluation of dairy goats in the United States and Canada. In: INTERNATIONAL CONFERENCE ON GOATS, 7, 2000, Poities. Proceedings... Poities, 2000 (CD-ROM).

SWALVE, H.H. The effect of test ay models on the estimation of genetic parametersand breeding values for dairy yields traits, Journal of Dairy Science, v.78, n.4, p.929-938, 1995.

THOLON, P.; QUEIROZ, S.A.; RIBEIRO, A.C. et al. Estudo genético quantitativo da produção de leite em caprinos da raça Saanen. Archivos Latinoamericanos Producción Animal, v.9, p. 1-5, 2001. 